\title{
Application of Orientation Imaging Microscopy in the TEM to studies of nanocrystalline materials
}

\author{
D. J. Dingley, M. J.Tiner, and S. I. Wright
}

TSL/EDAX, 392 E. 12300S. Draper, UT 40057

Orientation imaging microscopy (OIM) [1] is a familiar tool used in the SEM for the mapping of crystal orientations. It utilizes the Electron Backscatter Diffraction technique to collect the basic crystallographic information. OIM images have recently been obtained in the TEM. However, the basic crystallographic data is obtained using a new technique referred to as Dark Field Diffraction, [2]. The principle is to successively image the entire area of interest under a set of known dark field conditions and extract the crystal orientation for each pixel by recording the conditions when a pixel brights-up. Each bright-up condition defines a diffracting vector $\rho \theta$, where $\theta$ is the beam tilt and $\rho$ the azimuthal angle. From the set of diffraction vectors the crystal orientation can be determined. A crystallographic map is constructed from the measured orientations of all pixels in the image. In practice, the electron beam is under computer control and directed onto the specimen in directions that fall on a conical surface as illustrated in figure 1. Usually, 90 or 180 dark field images are recorded at equal intervals around the cone. Up to 40 such sets are recorded with the semi-cone angle increasing from just less than twice the Bragg angle for the lowest order reflection in the zero order Laue zone to greater than the angle needed to include diffraction from the first order. This is typically $0.5^{\circ}$ to $2.5^{\circ}$. The diffraction vectors effective for each pixel are extracted off-line from the set of dark field images by constructing a polar plot of the measured intensity. The radial axis is $\theta$ and the circumferential axis is $\rho$. An example plot is shown on figure 2 and is seen, in effect, to be a reconstruction of what the spot diffraction pattern from that pixel would have looked like in normal diffraction mode. The diffraction vectors are measured from the origin to the brightest point in the center of each spot. To improve accuracy in orientation measurement the vectors are fitted to the surface of the Ewald sphere, [3]. Greater than $1^{\circ}$ accuracy can be obtained in this way.

The set of measured orientations are used to draw grain and crystal orientation maps and to determine grain size and grain boundary types. Two examples are included here. Figure $3 \mathrm{a}$ is a bright field micrograph of $\mathrm{Cu}$ interconnect lines in a damascene prepared sample. Figure $3 \mathrm{~b}$ shows a grain map generated from the dark field diffraction data. The resolution is $5 \mathrm{~nm}$. Figures $4 \mathrm{a}$ and $4 \mathrm{~b}$ are orientation maps for a $95 \%$ deformed FeNi alloy. In figure 4a the coloring is designed to reveal individual grains and sub-grains in which internal structure is contiguous within $5^{\circ}$ rotation. In figure $4 \mathrm{~b}$ the coloring depicts the crystal direction normal to the surface. The key is inset. Figures $4 \mathrm{c}$ and $4 \mathrm{~d}$ show respectively the 111-pole figure and the inverse pole figure for the sample. The peak intensity, colored red, is 5 times random.

\section{References}

1. B.L.Adams, S.I.Wright and K Kunze,. Met. Trans. A 24(4) (1993) 819.

2. D.J.Dingley, S.I.Wright and D.J.Dingley Jr., MRS Symp. Hahzelden,C Hetherington and F.Ross eds. Materials Research Society. 253 (1998) 253.

3. S.Zaefferer, J.Appl.Cryst. 33 (2000) 10

4. The FeNi sample was supplied by Prof. R. Penelle and Dr T Baudin of University of Paris. 


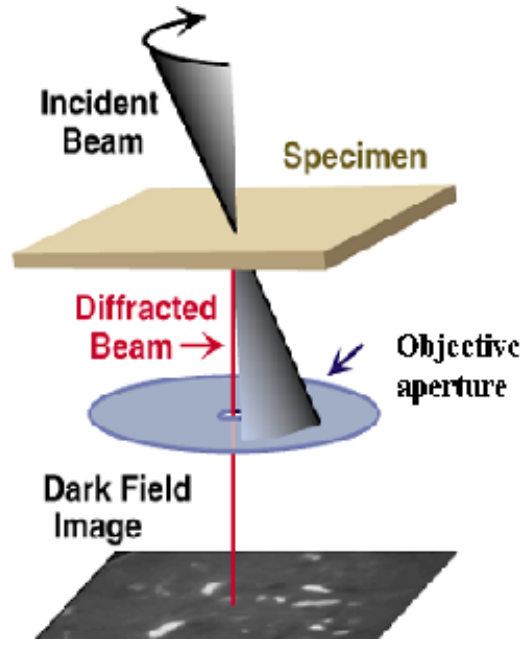

Figure 1. Schematic of conical scan mode employed in dark field diffraction technique

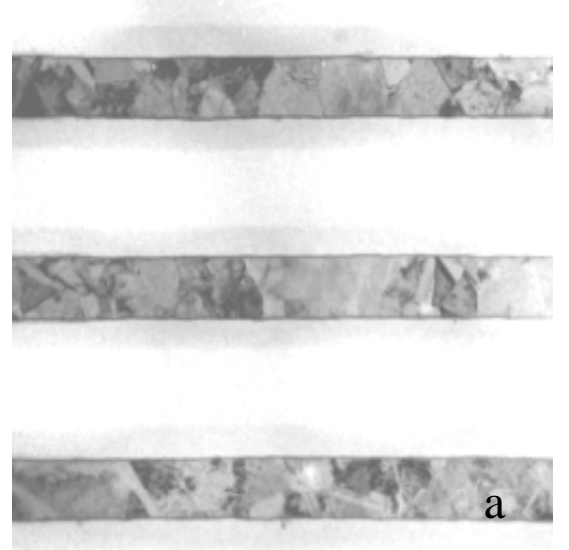

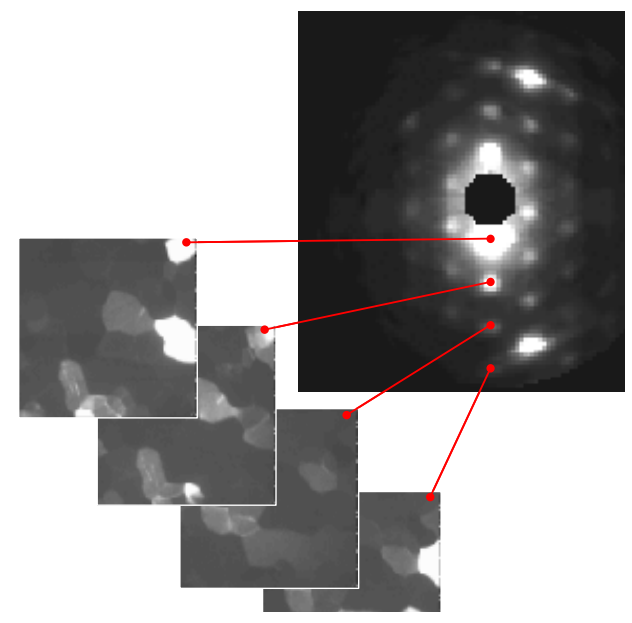

Figure 2. Construction of diffraction pattern from dark field images. Intensity of the marked pixel is mapped into the polar diagram

Figure3. (a) Bright field micrograph of $\mathrm{Cu}$ interconnect lines. (b) Grain map constructed from diffraction data.
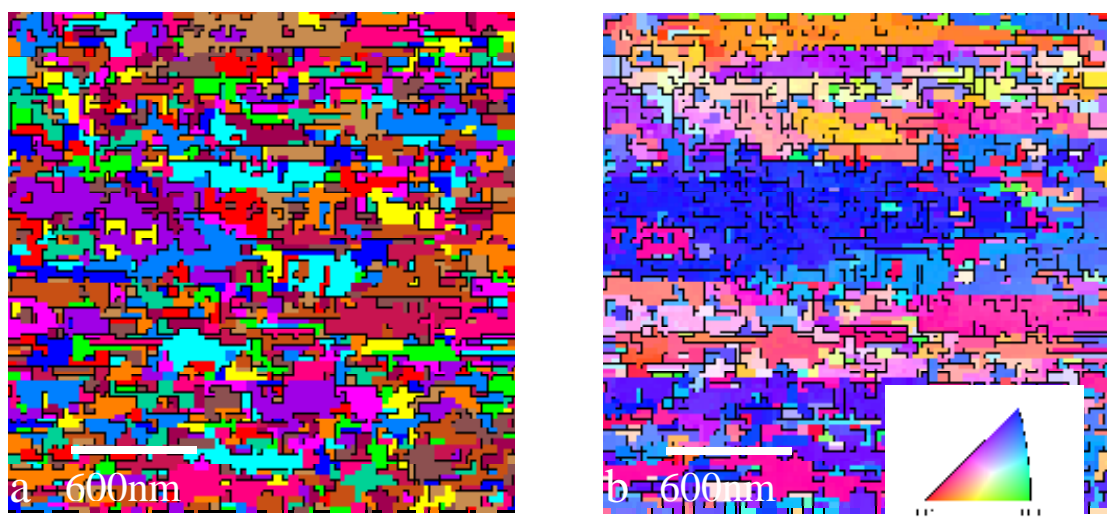
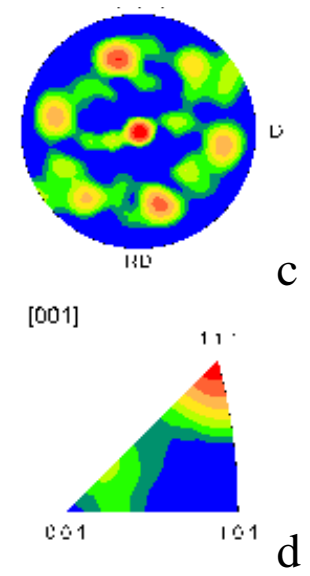

Figure 4. (a) Grain map of deformed FeNi alloy. (b) Crystal direction map. (c) 111 pole figure. (d) Inverse pole figure for surface normal. 